\title{
MICROLEAKAGE OF THREE CONTEMPORARY BULK FILL RESIN COMPOSITES; THERMOVISCOUS PREHEATED, SONIC FILL AND FLOWABLE BULK FILL IN CLASS I CAVITIES: IN VITRO STUDY
}

\author{
Mai Mamdouh M. Alsayed Akah*
}

\begin{abstract}
Objective: The aim was to compare the microleakage of three different types of bulk fill composites in class I cavities.

Materials and methods: Fifteen freshly extracted molars were selected for this study. Standardized occlusal cavities measuring $5 \mathrm{X} 4 \mathrm{X} 4 \mathrm{~mm}$ were prepared. The cavities were distributed among the three tested groups according to the type of resin composite; whereas; Group A: Viscalor thermoviscous preheated bulk fill, group B: Sonic fill bulk fill composite and group C: SUREFIL $\mathrm{SDR}^{\mathrm{TM}}$ flowable bulk fill. The restored molars were subjected to artificial aging, then teeth were immersed in $2 \%$ methylene blue dye solution for 24 hours at $37^{\circ} \mathrm{C}$. Each tooth was then sectioned into 2 halves and the specimens were examined under a stereomicroscope. All the specimens' images were subjected to image analysis to determine the depth of dye penetration in Mm. Statistical analysis was performed with IBM® SPSS $®$ Statistics Version 20 for Windows.
\end{abstract}

Results: A statistically significant difference was found between group B and each of group A and group $\mathrm{C}$ where $(p=0.018)$ and $(\mathrm{p}<0.001)$ respectively. No statistically difference was found between group $\mathrm{A}$ and group $\mathrm{C}$ where $(p=0.332)$. The highest mean value of dye penetration was found in group $\mathrm{B}$, while the least mean value was found in group $\mathrm{C}$.

Conclusions: None of the tested composites could completely eliminate microleakage along the tooth restoration interface in class I cavities. Flowable bulk fill composites and preheated composites showed less microleakage in comparison to the sonic fill composite.

KEYWORDS: Bulk fill composite, preheating, sonic activated, microleakage.

\footnotetext{
* Lecturer of Conservative Dentistry, Faculty of Dentistry, Cairo University Egypt.
} 


\section{INTRODUCTION}

Resin composite is the most frequently used direct restorative material for posterior restoration, because of the increase in patients' esthetics demands and the merits of tooth structure preservation. Despite the continuous improvements of the materials and techniques, polymerization shrinkage of resin composite is still considered the main problem. The generated shrinkage stresses result in microleakage at the restoration tooth interfaces ${ }^{1}$.

Microleakage is characterized by the invasion of microorganisms and their products, ions, and chemicals at the tooth restoration interface with subsequent consequences such as; postoperative sensitivity, marginal staining, recurrent decay, and eventually failure of restoration ${ }^{2}$.

Different attempts have been proposed to minimize the influence of polymerization shrinkage such as; increasing the filler load, modifying the monomer matrix, using different curing protocols, and incremental packing techniques ${ }^{3}$. Although, Incremental packing has been proven to minimize the overall volumetric shrinkage of resin composite especially in cavities with high $\mathrm{C}$-factor. It showed many adverse effects such as void incorporating, contamination between the subsequent layers as well as prolonged application times ${ }^{4}$.

In an attempt to simplify the packing technique and to shorten the chairside time, bulk fill composites were introduced ${ }^{5}$. Bulk-fill composites have gained widespread clinical use thanks to their improved curing properties, better control of polymerization shrinkage stresses and reduction of cuspal deflection ${ }^{6,7}$. Nowadays it is considered an acceptable alternative to conventional resin composites as proved by the systematic review and meta-analysis published by Mai Akah et al., in $2016^{8}$. Many types of bulk-fill composites were introduced having different insertion techniques. The technique by which the composite is introduced into the cavity affects the physical and mechanical properties of the restorative material. Unfortunately, internal voids are inevitable at the tooth restoration interface, the amount of which can be affected by the material type and the insertion technique.

Thermal energy increases the mobility of the molecular chains of the resin monomer and considerably enhances the flowability of pastelike composites. Also, ultrasonic energy yields a more random and looser configuration that results in the decreased viscosity of the composite upon extrusion ${ }^{9}$.

Many studies have evaluated the internal gap formation of dental resin composites ${ }^{10,11}$. However, little data is available regarding the effects of different insertion techniques of the contemporary bulk fill composites on the microleakage and internal gap formation to cavity walls and margins.

Therefore, the purpose of this investigation was to compare the microleakage of three different types of bulk fill composites with different insertion techniques; Viscalor (thermoviscous preheated), sonic fill (sonic activated), and SUREFIL SDR ${ }^{\mathrm{TM}}$ (Smart Dentin Replacement flowable bulk) in class I cavities assessed with dye penetration. The null hypothesis was that there would be no difference in the microleakage between the tested composites.

\section{MATERIALS AND METHODS}

\section{Tooth selection, grouping and cavity preparation}

Fifteen freshly extracted, caries-free human molars were selected for this study. The molars were randomly distributed among the three tested groups according to the type of resin composite $(n=5$ molars); whereas; Group A: Viscalor thermoviscous preheated bulk fill, group B: sonic fill bulk fill composite, and group C: SUREFIL SDR ${ }^{\mathrm{TM}}$ flowable bulk fill composite.

Standardized occlusal cavity measuring $5 \mathrm{~mm}$ in mesiodistal diameter, $4 \mathrm{~mm}$ in buccolingual diameter, and $4 \mathrm{~mm}$ in depth was prepared in each 
tooth. Cavities were prepared using diamond tips (SF-31SC, Mani, INC, Japan) attached to a highspeed air turbine under water cooling. The bur was replaced after every five preparations. The cavity dimensions were verified with a periodontal probe (figure 1).

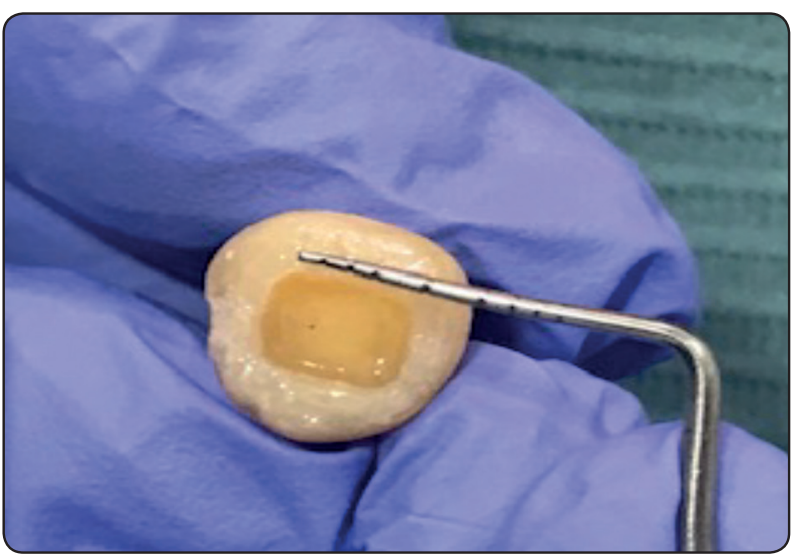

Fig. (1): The cavity dimensions were verified with a periodontal probe.

\section{Bonding procedure}

The bonding procedure was done for all the cavities using the universal adhesive; All bond universal (Bisco, Schaumburg, IL, USA) following the selective enamel etching technique. Whereas; the cavity enamel margins were etched for 30 seconds, thoroughly rinsed for 20 seconds, and dried. Then the bonding agent was applied into the cavity walls in two consecutive coats with 15 seconds of air blowing for each, and light-cured once for 20 seconds. The light cure device used in this study was the Bluephase light-curing unit (Ivoclar Vivadent, Schaan, Liechtenstein).

\section{Restorative procedures}

For group A: The viscalor composite compule (A2) was loaded into the Viscalor dispenser and heated on mode 1 for 30 seconds heating time to a temperature of $65^{\circ} \mathrm{C}$. After the preheating time, the heating device stopped indicating that the compule reached the required temperature. The dispenser light flushed, indicating the start of the packing time
(2.5 minutes) as per the manufacturer's instructions. And the composite was then packed into the cavity in a single increment of $4 \mathrm{~mm}$ depth and light-cured for 20 seconds (figure 2).

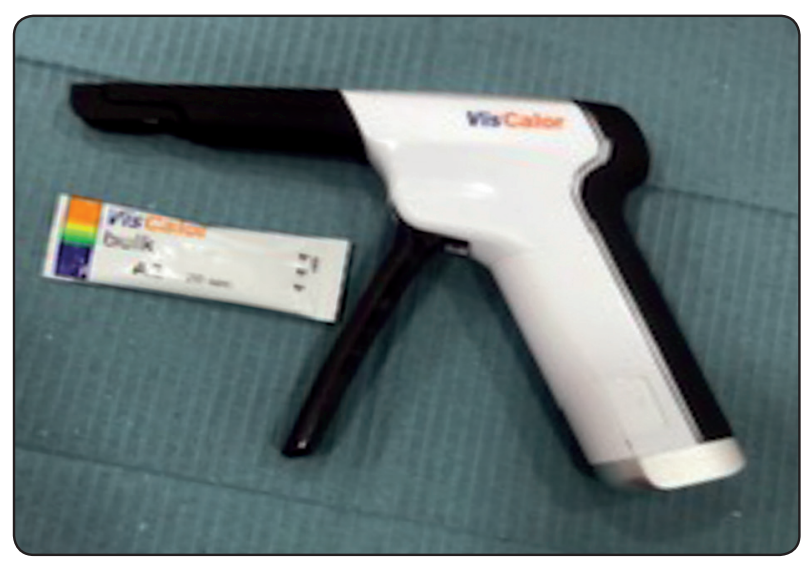

Fig. (2): Viscalor dispenser and composite compule.

For group B: The sonic fill composite compule (A2) was uncapped and loaded into the sonic fill 2 handpiece and attached to the dental air turbine and the sonic energy level was adjusted to 3 . The resin composite was then inserted into the cavity in a single increment of $4 \mathrm{~mm}$ depth, and light-cured for 20 seconds (figure $\mathbf{3}$ ).

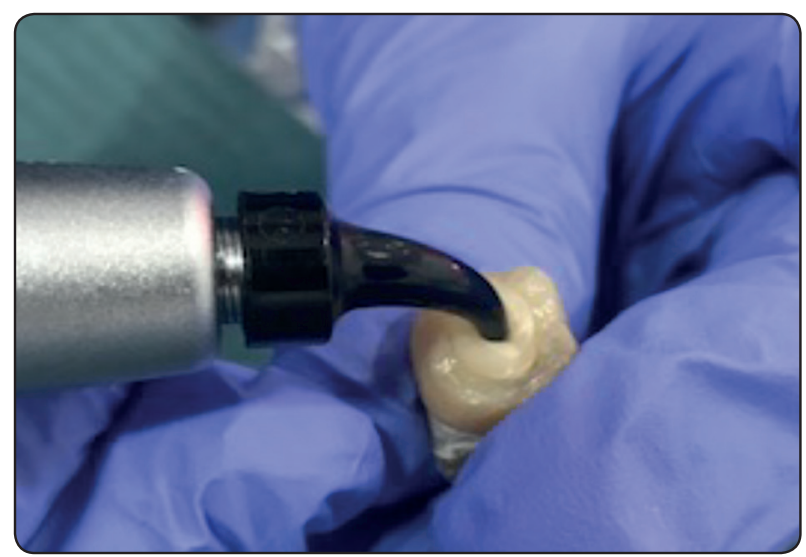

Fig. (3): Sonic fill composite application.

For group C: The flowable bulk fill composite SureFil SDR was injected in a $3 \mathrm{~mm}$ bulk increment to fill the dentinal part of the cavity, then lightcured for 20 seconds. As per the manufacturer's 
instructions, the top one $\mathrm{mm}$ of the cavity was restored using the nanoceramic composite Ceram $\mathrm{X}$ Mono (A2) that was also light-cured for 20 seconds (figure 4). The teeth were stored in distilled water at temperature $37^{\circ} \mathrm{C}$ for 24 hours before the artificial aging.

The material types, insertion techniques, composition, and manufacturers are presented in table 1.

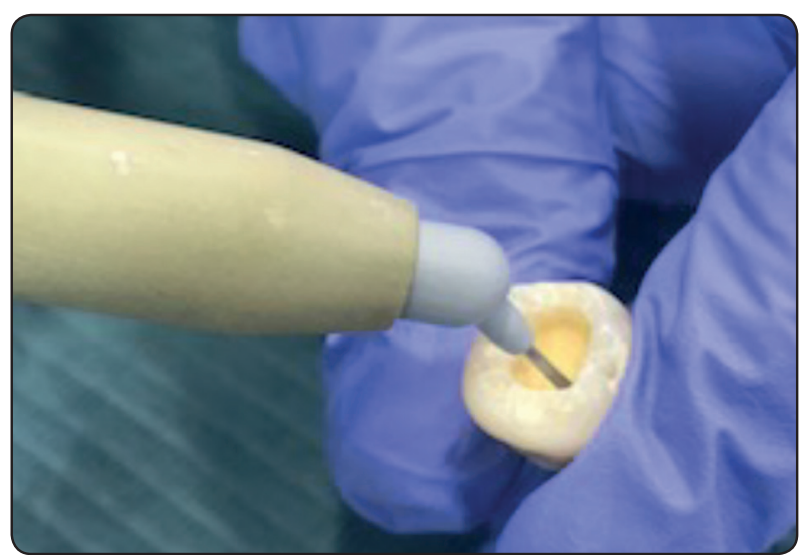

Fig. (4): SureFil SDR composite application.

TABLE (1): Types, insertion technique, composition and manufacturers of the materials used in this study

\begin{tabular}{|c|c|c|c|c|c|c|}
\hline Product & Material type & $\begin{array}{l}\text { Insertion } \\
\text { technique }\end{array}$ & Shade & Matrix composition & $\begin{array}{l}\text { Filler \% } \\
\text { by weight }\end{array}$ & Manufacturer \\
\hline Viscalor & $\begin{array}{l}\text { Thermoviscous bulk } \\
\text { fill paste like resin } \\
\text { composite }\end{array}$ & Heated & A2 & $\begin{array}{l}\text { Bis-GMA, } \\
\text { dimethacrylate, } \\
\text { fillers }\end{array}$ & 83 & $\begin{array}{l}\text { VOCO, Cuxhaven, } \\
\text { Germany } \\
\text { www.voco.dental.com }\end{array}$ \\
\hline Sonic fill 2 & $\begin{array}{l}\text { Bulk-fill paste-like } \\
\text { resin composite }\end{array}$ & $\begin{array}{l}\mathrm{S} \text { o n i c } \\
\text { activated }\end{array}$ & $\mathrm{A} 2$ & 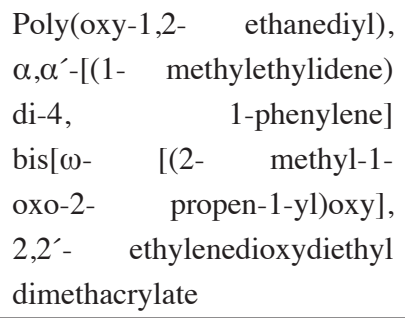 & 81.3 & $\begin{array}{l}\text { Kerr, CA, Orange, } \\
\text { USA } \\
\text { www.kerrdental.com }\end{array}$ \\
\hline $\begin{array}{l}\text { S UREFIL } \\
\text { SDR }^{\mathrm{TM}}\end{array}$ & $\begin{array}{l}\text { Flowable bulk fill } \\
\text { resin composite }\end{array}$ & Injectable & $\mathrm{A} 2$ & $\begin{array}{l}\text { Barium and strontium } \\
\text { alumino-fluoro-silicate glass, } \\
\text { TEGDMA, modified UDMA, } \\
\text { DMA, EBPADMA, pigment, } \\
\text { photoinitiator }\end{array}$ & 68 & $\begin{array}{l}\text { Dentsply Caulk, } \\
\text { Milford, DE, USA/ } \\
\text { www.dentsplysirona. } \\
\text { com }\end{array}$ \\
\hline $\begin{array}{l}\text { Cera m. X } \\
\text { Mono }\end{array}$ & $\begin{array}{l}\mathrm{N} \text { a n o-c e r a m i c } \\
\text { composite }\end{array}$ & Packable & A2 & $\begin{array}{l}\text { Methacrylate modified } \\
\text { polysiloxane, DMA, barium- } \\
\text { aluminum-borosilicate glass, } \\
\text { methacrylate functionalized } \\
\text { silicon dioxide nanofillers }\end{array}$ & 76 & \\
\hline $\begin{array}{l}\text { Scotchbond } \\
\text { univers a l } \\
\text { etchant }\end{array}$ & $\begin{array}{l}35 \% \text { phosphoric acid } \\
\text { gel }\end{array}$ & NA & NA & NA & NA & $\begin{array}{l}\text { 3M, St Paul, MN, } \\
\text { USA } \\
\text { www.3M.com }\end{array}$ \\
\hline $\begin{array}{l}\text { All Bond } \\
\text { Universal }\end{array}$ & $\begin{array}{l}\text { Universal light cure } \\
\text { adhesive system }\end{array}$ & NA & NA & $\begin{array}{l}\text { MDP, Bis-GMA, HEMA, } \\
\text { ethanol, and water. }\end{array}$ & - & $\begin{array}{l}\text { Bisco, Schaumburg, } \\
\text { IL, USA } \\
\text { www.bisco.com }\end{array}$ \\
\hline
\end{tabular}

Abbreviations: Bis-GMA; bisphenol-A diglycidyl ether dimethacrylate; TEGDMA; triethylene glycol dimethacrylate, UDMA; urethane dimethacrylate, DMA; dimethacrylate, EBPADMA; ethoxylated bisphenol A dimethacrylate, MDP; 10-methacryloyloxydecyl dihydrogen phosphate, HEMA; hydroxyethyl methacrylate. 


\section{Artificial aging, dye Penetration, and specimen preparation}

The restored molars were subjected to artificial aging by thermocycling for 1000 cycles between temperatures of $5^{\circ} \mathrm{C}$ and $55^{\circ} \mathrm{C}$, with a dwell time of 30 seconds in each bath and a transfer time of 10 seconds $^{12}$ in the 100 SD Mechatronic thermocycler, Germany.

Double coats of nail varnish were applied on the tooth surfaces, except for $1 \mathrm{~mm}$ away from the restoration margins. Afterward, the teeth were immersed in $2 \%$ methylene blue dye solution (Imperial Chemical Industries, London, England) for 24 hours at $37^{\circ} \mathrm{C}$, and then thoroughly rinsed for five minutes ${ }^{13}$. Each tooth was then sectioned mesiodistally into 2 halves with a micro slicing machine (IsoMet 4000; Buelher, USA) to result in ten specimens per group $(n=10)$ (figure 5).

\section{Interface examination and Microleakage mea- surement (image analysis):}

The specimens were then examined under a stereomicroscope (Nikon MA 100, Japan) at a magnification of X50 to determine the extent of dye penetration along with the tooth restoration interface. All the specimens' images were subjected to image analysis to determine the depth of dye penetration in Mm using the image analysis software (Omnimet, Buehler, Germany) (figure 6) .

\section{Statistical analysis:}

The mean and standard deviation values for the dye penetration depth were calculated for each group. Data were explored for normality using Kolmogorov-Smirnov and Shapiro-Wilk tests and showed parametric (normal) distribution. One-way ANOVA followed by Tukey post hoc test was used to compare between more than two groups in nonrelated samples. The significance level was set at $\mathrm{P} \leq 0.05$. Statistical analysis was performed with IBM ${ }^{\circledR}$ SPSS ${ }^{\circledR}$ Statistics Version 20 for Windows.

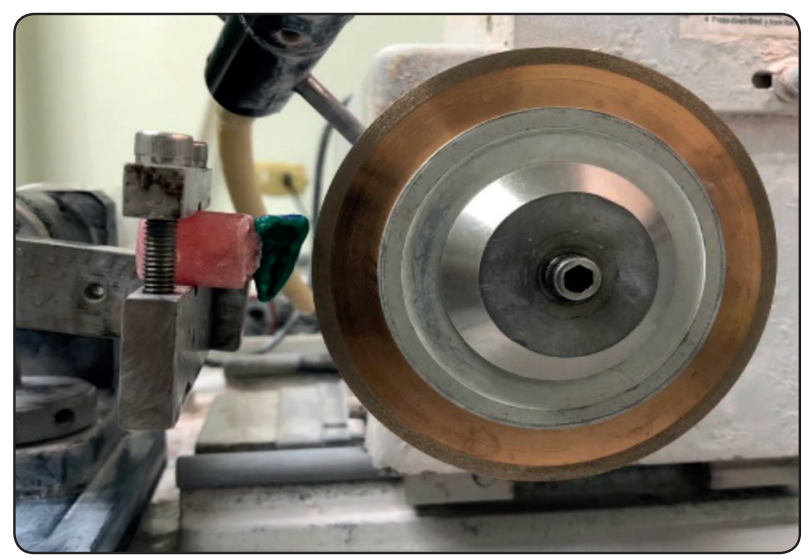

Fig. (5): Tooth sectioning into two halves using a micro slicing machine.

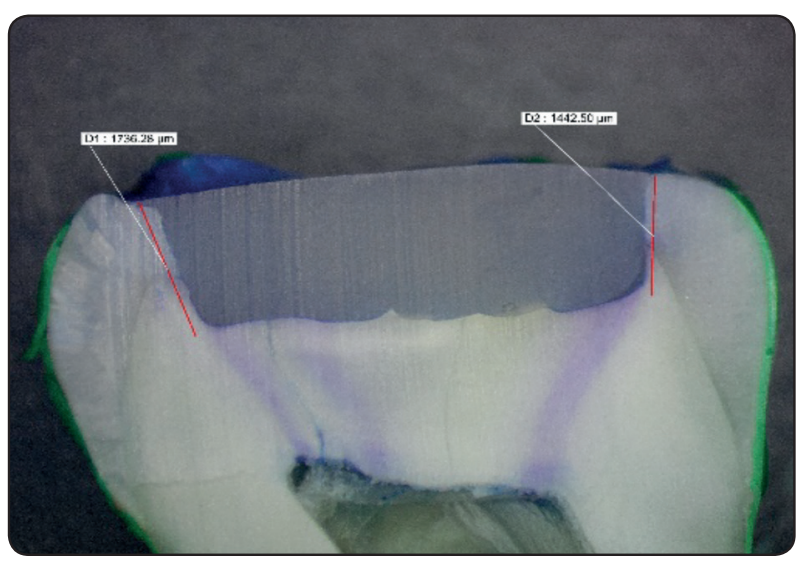

Fig. (6): Determining the depth of dye penetration using the image analysis software.

\section{RESULTS}

The means and standard deviations of the dye penetration depth along with the tooth restoration interface for the three tested composites are presented in table 2 and figure 7. There was a statistically significant difference between group A, group B, and group $\mathrm{C}(p=0.001)$. A statistically significant difference was found between group $B$ and each of groups A and group C, where $(p=0.018)$ and $(p<0.001)$, respectively. No statistically significant difference was found between group A and group $\mathrm{C}$ where $(p=0.332)$. The highest mean value for dye penetration was found in group B, while the least mean value was found in group $\mathrm{C}$. 
TABLE (2): The mean, standard deviation (SD) values of penetration depth of different groups.

\begin{tabular}{|c|c|c|}
\hline \multirow{2}{*}{ Variables } & \multicolumn{2}{|c|}{ Penetration depth } \\
\hline & Mean & SD \\
\hline $\begin{array}{c}\text { Group A } \\
\text { Viscalor thermoviscous }\end{array}$ & $1572.79^{b}$ & 209.16 \\
\hline $\begin{array}{l}\text { Group B } \\
\text { Sonic fill } 2\end{array}$ & $1795.95^{\mathrm{a}}$ & 66.32 \\
\hline $\begin{array}{c}\text { Group C } \\
\text { SDR flowable bulk fill }\end{array}$ & $1462.87^{b}$ & 195.89 \\
\hline
\end{tabular}

*; significant $(p<0.05) \quad n s ;$ non-significant $(p>0.05)$

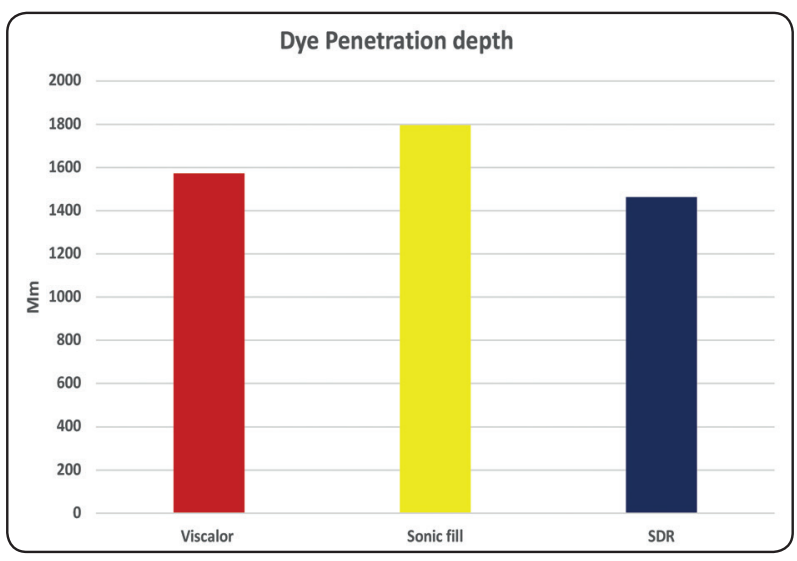

Fig. (7): Bar chart representing the dye penetration depth in $\mathrm{Mm}$ for the three tested composites.

\section{DISCUSSION}

The study aimed to compare the effect of different insertion techniques of three types of bulk fill resin composites on the microleakage in class I cavities after artificial aging using the dye penetration technique. The results showed a significant difference in the microleakage degrees amongst the three tested bulk fill composites. Every resin composite type has a different insertion technique, emphasizing that the material type and placement technique influence the internal adaptation to cavity walls and margins. Thus, the null hypothesis was rejected.
Bulk fill composites can fill the cavity in a single layer making the procedure less technique sensitive $^{8}$. Additionally, Bulk fill formulations allow for modulation of the polymerization reaction by using stress-relieving monomers and fillers and the incorporation of reactive photo-initiators. Furthermore, bulk placement reduces void incorporation and contamination between composite layers, leading to more durable restorations ${ }^{14,15}$.

Similar to conventional composites, filler types and contents, matrix compositions and manufacturing process of bulk fill resin composites are quite different between the different categories either; preheated, sonic activated or flowable bulk fill composites $^{16,17,18}$.

Moreover, the viscosity of the composite material affects the adaptability and hence the gap formation incidence between the composite and the cavity walls and margins. Such gaps severely affect the mechanical properties of the composite material, particularly under aging conditions, since they could act as stress raisers, and lead to clinical failures of the restorations ${ }^{19,20,21}$.

Composite preheating is an alternative method to the conventional placement of resin composites. Composite heating reduces the material viscosity without affecting the composition and physicochemical properties, which allows for improving composite to cavity wall adaptation with the subsequent reducing internal voids. ${ }^{22,23}$. The Viscalor dispenser is a newly introduced heating device to be used in combination with Viscalor bulk fill composite compules. The manufacturer claims that the dispenser application gives the material a lower viscosity, allowing it to flow optimally into the cavity margins and undercuts, minimizing the chance for air bubbles entrapment and the risk of marginal gaps. The device mode 1 is heating to a temperature of $65^{\circ} \mathrm{C}$ for 30 seconds pre-warming time and 2.5 minutes working time. ${ }^{24}$

The sonic activated sonic fill composite resin has a depth of cure of $5 \mathrm{~mm}$. It has a specific handpiece 
that provides sonic energy at different intensities. As the sonic energy is generated through the handpiece, the built-in modifier causes the viscosity to decrease up to $87 \%{ }^{25}$.

Also, Flowable bulk fill composites are an alternative option used to fill the deeper aspects of the cavity with its flowable consistency enhancing the material adaptation and self-leveling within the cavity confinement. It can fill up to 4-6 mm depth with the addition of a conventional resin composite occlusally ${ }^{26}$.

Class I cavity design was selected in this study, so that, it was possible to assess the degree of leakage under such challenging stresses generated at the highest $\mathrm{C}$-factor in comparison to any compound or complex cavity designs ${ }^{27}$. A single type of universal adhesive system was selected to bond all the resin composite types, to standardize the impact of the adhesive system and adhesion protocol on the microleakage of the three tested composites.

The dye penetration technique is one of the most commonly used methods for microleakage assessment in vitro $^{28}$. This technique is highly feasible with no radiation hazards. In addition, the dye has a contrasting color to both the tooth and the restoration, without any chemical interactions with the specimens. Thermal cycling was applied to the samples, simulating the degradation of the bond interface that occurs in the oral cavity ${ }^{29,30}$. The results of the current study revealed a statistical difference between the sonic fill technique and each of the Viscalor and the SDR, with more leakage reported in the sonic fill group. This result was in an agreement with Eunice et al., 2012, who found no effect for the sonic application in minimizing the microleakage to cavity walls ${ }^{31}$. Also, Julio et al., 2020, who showed higher interfacial stress and lower bond strength for sonic fill composite with dentin in comparison to Tetric N-Ceram and Tetric $\mathrm{N}$-Ceram bulk fill. Their result could be attributed to the low modulus of elasticity together with the higher tensile and compressive strength of sonic fill composite (8.6 GPa). Despite this interaction of mechanical properties reduced the internal stresses inside the material, it raised the interfacial stresses at the cavity walls and margins ${ }^{26}$.

The results were also following Gulbike et al., 2020, who observed increasing void percentage for sonic fill packing compared to conventional and preheated composites ${ }^{32}$. This result was attributed to the sonic vibration, which resulted in more air intake into the restoration. Furthermore, sonication may lead to the gathering of smaller and isolated bubbles already present in the resin into larger void sizes. It was also found that when the sonicated composite returns to its original rheological properties, the air trapped in the material becomes more pronounced and could severely affect the restoration properties ${ }^{33}$.

In addition, the restorative procedure using the sonic fill system is more difficult, and it requires a trained operator for better control over the ultrasonic device $^{34}$.

Regarding the increased leakage in the sonic fill group in comparison to the other types, this could be attributed to the degree and the rate of viscosity decrease. As previously mentioned, sonication decreased the viscosity up to $87 \%$ during the composite insertion and when the sonic energy is interrupted, the composite resin returns to a more viscous consistency, suitable for sculpturing. This is not happening with the flowable bulk fills which retain their low viscosity and hence keep the adaptation to cavity walls and margins ${ }^{26}$. The superior adaptation of Viscalor preheated composite is justified in literature by the thermal energy that forces the composite monomers apart, allowing them to slide by each other more readily improving the material adaptation. Additionally, the rate of cooling of the Viscalor composite which is about 3 minutes is a relatively long period permitting suitable adaptation to cavity walls together with better stress release if compared to the sonic fill resin composite ${ }^{24,35}$. Another study emphasized the effect of composite heating on material viscosity by testing the viscosity of bulk fill composites 
at different temperatures. The results revealed a viscosity of $0.05 \pm 0.00 \mathrm{kPa} s$ at $25^{\circ} \mathrm{C}$ and $0.03 \pm 0.01$ $\mathrm{kPa} \mathrm{s}$ at $37^{\circ} \mathrm{C}$; which was about $48.7 \%$ reduction in viscosity with heating ${ }^{36}$. The results of this study could be correlated to another study done by Yang et al., in 2019, which evaluated the composite extrusion force. They found that the extrusion force required for the preheated Viscalor compule was $66.49 \pm 14.16 \mathrm{~N}$, while, $153.62 \pm 1.56 \mathrm{~N}$ force was required to extrude the unheated compule ${ }^{37}$, indicating a pronounced decrease in the material's viscosity with the Viscalor heating.

SDR flowable bulk fill showed the least microleakage levels in comparison to the other types. This result was in agreement with another study that tested the marginal sealing ability of flowable bulk fill composite versus conventional composite to enamel and dentin. Although no difference was found for the enamel, bulk fill resins showed better marginal seal with dentin ${ }^{38}$. This was explained by the intimate union between the flowable bulk fill composite with the cavity floor and walls. Moreover, flowable bulk acted as a flexible intermediate layer that helped in the release of shrinkage stresses. Also, the increased translucency of SDR allows for deeper light transmission as well as the incorporation of modified UDMA in the composite matrix are considered the causes behind the stress releasing property of the SDR. The SDR technology also comprises the unique polymerization modulator which is embedded at the center of the resin. Those modulators resulted in a higher depth of cure and decreased interfacial gap together with the self-leveling property ${ }^{39,40}$. In addition to the inherent properties of the SDR flowable bulk fill composite, the dye penetration might be decreased because of the sealability of the overlying nanoceramic composite. This top coating layer might help to prevent the dye from penetrating deeper along with the underneath SDR- dentin interface.

Possible limitations of the study are the use of a single energy level in the sonic fill handpiece (Level 3 ) and a single heating temperature $\left(65^{\circ} \mathrm{C}\right)$. Different sonic frequencies, as well as different tempera- tures, might have different effects on the physical and rheological properties of bulk fill composites and hence on their microleakage. Moreover, further studies are required to validate the clinical performance of these bulk fill resins in vivo.

\section{CONCLUSIONS}

Based on the study findings, and within the limitations of an in vitro study the following conclusions can be drawn:

- None of the evaluated composite categories could completely prevent microleakage along with the tooth restoration interface in class I cavities.

- Flowable bulk fill composites and preheated composites showed better adaptation to the cavity walls in comparison to the sonic fill composite.

- Sonic fill composite packing is a less predictable technique requiring training and experience in comparison to the other tested techniques.

\section{ACKNOWLEDGMENT}

Deep gratitude goes to Professor Ahmed Elzoheiry, Professor of Conservative Dentistry, Cairo University for supplying the Viscalor dispenser device used in this study.

\section{REFERENCES}

1. McHugh L, Politi I, Al-Fodeh R, Fleming G. Implications of resin-based composite $(\mathrm{RBC})$ restoration on cuspal deflection and microleakage score in molar teeth: Placement protocol and restorative material. Dental Materials 2017; 33: 329-335.

2. Alani A, Toh C. Detection of microleakage around dental restorations: a review. Operative Dentistry 1997; 22: 173-185.

3. Ferracane J. Buonocore Lecture. Placing dental composites- a stressful experience. Operative Dentistry 2008; 33 : 247-257.

4. El-Safty S, Silikas N, Watts D. Creep deformation of restorative resin-composites intended for bulk-fill placement. Dental Materials 2012; 28: 928-935. 
5. Roggendorf MJ, Kramer N, Appelt A, Naumann M, Frankenberger R. Marginal quality of flowable 4-mm base vs. conventionally layered resin composite. Journal of Dentistry 2011; 39: 643-647.

6. Algamaiah H, Sampaio C, Rigo L, Janal M, Giannini M, Bonfante E. Microcomputed tomography evaluation of volumetric shrinkage of bulk-fill composites in class II cavities. Journal of Esthetic Restorative Dentistry 2017; 29: 118-127.

7. El-Damanhoury H, Platt J. Polymerization shrinkage stress kinetics and related properties of bulk-fill resin composites. Operative Dentistry 2014; 39: 374-382.

8. Mamdouh M, Daifalla L, Yousry M. Bonding of Bulk Fill versus Contemporary Resin Composites: A Systematic Review and Meta-analysis. Indian journal of Science and Technology 2017; 9 (20): 1-11.

9. Furness A, Tadros M, Looney S, Rueggeberg F. Effect of bulk/incremental fill on internal gap formation of bulk-fill composites. Journal of Dentistry 2014; 42: 439-449.

10. Demirel G, Baltacioglu I, Kolsuz M, Ocak M, Orhan K. Micro-computed tomography evaluation of internal void formation of bulk-fill resin composites in Class II restorations. Polymers Composium 2019; 40: 2984-2992.

11. Mehdikhani M, Gorbatikh L, Verpoest I, Lomov S. Voids in fiber-reinforced polymer composites: A review on their formation, characteristics, and effects on mechanical performance. Journal of Composite Materials 2018; 53: 1579-1669.

12. Bibhu D, Pallavi S, Munmun M, Vikas S, Ajay K, Dhruv G. In vitro Evaluation and Comparison of Microleakage of Two Restorative Composite Resins in Class II Situations using Confocal Laser Scanning Microscopy. Journal of Contemporary Dental Practice 2018;19 (9):1100-1104.

13. Kalmowicz J, Phebus J, Owens B, Johnson W, King G. Microleakage of Class I and II Composite Resin Restorations Using a Sonic-resin Placement System. Operative Dentistry 2015; 40 (6): 653-66.

14. Chuang S, Liu J, Chao C, Liao F, Chen Y. Effects of flowable composite lining and operator experience on microleakage and internal voids in class II composite restorations. Journal of Prosthetic Dentistry 2001; 85 (2):177- 183. 36.

15. Par M, Gamulin O, Marovik D, Klaric E, Tarle Z. Raman spectroscopic assessment of degree of conversion of bulkfill resin composites - changes at 24 hours post cure. Operative Dentistry 2015; 40 (3):E92-101.
16. Olmez A, Oztas N, Bodur H. The effect of flowable resin composite on microleakage and internal voids in class II composite restorations. Operative Dentistry 2004; 29: 713-719.

17. De Gee A. Some aspects of vacuum mixing of composite resins and its effect on porosity. Quintessence International Dental Digest 1979; 10: 69-74.

18. Jorgensen K, Hisamitsu H. Porosity in microfill restorative composites cured by visible light. Scandinavian Journal of Dental Research 1983; 91: 396-405.

19. Van Ende A, De Munck J, Lise DP, Van Meerbeek B. Bulkfill composites: A review of the current literature. Journal of Adhesive Dentistry 2017; 19: 95-109.

20. Chuang S, Liu J, Chao C, Liao F, Chen Y. Effects of flowable composite lining and operator experience on microleakage and internal voids in class II composite restorations. Journal of Prosthetic Dentistry 2001; 85: 177-183.

21. Olmez A, Oztas N, Bodur H. The effect of flowable resin composite on microleakage and internal voids in class II composite restorations. Operative Dentistry 2004; 29: 713-719.

22. Deb S, Di Silvio L, Mackler H, Millar B. Pre-warming of dental composites. Dental Materials 2011; 27: e51-59.

23. Taubock T, Tarle Z, Marovic D, Attin T. Pre-heating of high viscosity bulk-fill resin composites: effects on shrinkage force and monomer conversion. Journal of Dentistry 2015; 43: 1358-1364.

24. Jiawei Y, Nikolaos S, David C. Pre-heating time and exposure duration: Effects on post-irradiation properties of a thermo-viscous resin-composite. Dental materials 2020; 36, 787-793.

25. Júlio C, Ana Rosa C, Crisnicaw V, Renata W, Saturnino C, Ana Maria S, Gilberto A, Lourenço C. Interfacial Stress and Bond Strength of Bulk-Fill or Conventional Composite Resins to Dentin in Class II Restorations. Brazilian Dental Journal 2020; 31(5): 532-539.

26. Scientific documentation of Tetric EvoCeram Bulk Fill. Retrieved online March, 2016 from http://www.ivoclarvivadent. Com

27. Kim R, Kim Y, Choi N, Lee I. Polymerization shrinkage, modulus, and shrinkage stress related to tooth-restoration interfacial debonding in bulk-fill composites. Journal of Dentistry 2015; 43: 430-439.

28. De Munck J, Van Landuyt K, Peumans M. A critical review of the durability of adhesion to tooth tissue: Methods and results. Journal of Dental Research 2005; 84:118-132. 
29. Yap A. Effects of storage, thermal and load cycling on a new reinforced glass-ionomer cement. Journal of Oral Rehabilitation 1998; 25: 40-44.

30. Gale M, Darvell B. Thermal cycling procedures for laboratory testing of dental restorations. Journal of Dentistry 1999; 27: 89-99.

31. Eunice C, Margarida A, João C. The evaluation of microleakage of composite resin restorations with Sonic fill. An in vitro experimental model. Open Journal of Stomatology 2012; 2: 340-347.

32. Gülbike D, Ayşe Işı1 O, Özgür I, Fulya A, Arda B, Burak B, Kaan O. Micro-computed tomographic evaluation of the effects of pre-heating and sonic delivery on the internal void formation of bulk-fill composites. Dental Materials 2020; 1-7.

33. Hirata R, Pacheco R, Caceres E, Janal M, Romero M, Giannini $\mathrm{M}$. Effect of sonic resin composite delivery on void formation assessed by micro-computed tomography. Operative Dentistry 2018; 43: 144-150.

34. Iovan G, Stoleriu S, Moldovanu A, Morogai S, Andrian S. SEM study of the interface between the cavity wall and composite resin in cavities filled using vibration. International Journal of Medical Dentistry 2011; 1: 254-258.

35. Blalock J, Holmes R, Rueggeberg F. Effect of temperature on unpolymerized composite resin film thickness. Journal of Prosthetic Dentistry 2006; 96: 424-32.

36. Al-Ahdal K, Silikas N, Watts DC. Rheological properties of resin composites according to variations in composition and temperature. Dental Materials 2014; 30: 517-524.

37. Yang J, Silikas N, Watts D. Pre-heating effects on extrusion force, stickiness and packability of resin-based composite. Dental Materials 2019; 35: 1594-1602.

38. Scotti N, Comba A, Gambino A, Paolino D, Alovisi M, Pasqualini. Microleakage at enamel and dentin margins with a bulk fills flowable resin. European Journal of Dentistry $2014 ; 8: 1-8$.

39. Zeynep K, Ayse Y. Microleakage evaluation of different types of bulkfill composites on enamel and cementum. Clinical Dentistry And Research 2019; 43(3): 100-109.

40. Orlowski M, Tarczydlo B, Chalas R. Evaluation of marginal integrity of four bulk-fill dental composite materials: in vitro study. Scientific World Journal 2015; 70-76. 